\title{
ON A SEQUENCE OF FOURIER COEFFICIENTS
}

\section{BASUDEO SINGH}

1. Let $f(t)$ be a function which is integrable in the sense of Lebesgue over the interval $(-\pi, \pi)$ and is defined outside this interval by periodicity. Let the Fourier series of $f(x)$ be

(1.1) $\frac{1}{2} a_{0}+\sum_{n=1}^{\infty}\left(a_{n} \cos n x+b_{n} \sin n x\right)=\frac{1}{2} a_{0}+\sum_{1}^{\infty} A_{n}(x)$;

then the conjugate series of (1.1) is

$$
\sum_{n=1}^{\infty}\left(b_{n} \cos n x-a_{n} \sin n x\right)=\sum_{1}^{\infty} B_{n}(x) .
$$

Fejèr $[3$, p. 62] has shown that if $l=f(x+0)-f(x-0)$ exists and is finite, the sequence $\left\{n B_{n}(x)\right\}$ is summable $(C, r), r>1$, to the value $l / \pi$; and if $f$ is of bounded variation, the theorem holds true for $r>0$. It has also been proved that if $l$ exists and is finite, the sequence $\left\{n B_{n}(x)\right\}$ is summable by the first logarithmic mean to the same value $[3$, p. 62$]$.

Obrechkoff [2] has shown that if $f$ is integrable $(L)$ and if $t^{-1} \mid f(x+t)$ $-f(x-t)-l \mid$ is integrable near $t=0$, then $n^{-1} \sum_{1}^{n} r B_{r}(x) \rightarrow \pi^{-1} l$.

Mohanty and Nanda [1] have proved that if

$$
f(x+t)-f(x-t)-l=o\left\{(\log 1 / t)^{-1}\right\} \quad \text { as } t \rightarrow 0
$$

and $a_{n}$ and $b_{n}$ are $O\left(n^{-\delta}\right), 0<\delta<1$, then the sequence $\left\{n B_{n}(x)\right\}$ is summable $(C, 1)$ to the value $l / \pi$. From this result they have deduced the Hardy and Littlewood's test for convergence of the conjugate series (1.2) by applying a Tauberian theorem of Hardy and Littlewood.

We write

$$
\psi(t)=f(x+t)-f(x-t)-l .
$$

The object of this note is to prove the following theorem:

THEOREM 1. If

$$
\Psi(t)=\int_{0}^{t} \psi(u) d u=o(t)
$$

and

Received by the editors January 20, 1955 and, in revised form, August 3, 1955. 


$$
\int_{\epsilon}^{\delta} \frac{|\psi(t+\epsilon)-\psi(t)|}{t} d t \rightarrow 0
$$

for some fixed $\delta$, when $\epsilon \rightarrow+0$, then the sequence $\left\{n B_{n}(x)\right\}$ is summable $(C, 1)$ to the value $l / \pi$.

From Theorem 1, we deduce Lebesgue's test for convergence of the conjugate series (1.2) by applying Tauber's Second Theorem.

It is to be noted that Obrechkoff has proved the summability $(C, 1)$ of the sequence $\left\{n B_{n}(x)\right\}$ under Dini's criterion for the convergence of Fourier series while from Fejèr's result it is obvious that the sequence is also summable $(C, 1)$ under Jordan's criterion. In Theorem 1, we prove the same result under Lebesgue's criterion. As it is well known that Lebesgue's test includes both Dini's and Jordan's tests, Theorem 1 includes the above mentioned results of Obrechkoff and Fejèr.

I am much indebted to Dr. M. L. Misra for his kind interest and advice in the preparation of this paper.

2. We shall require the following lemmas:

LEMMA 1. If

$$
I=\int_{\pi / n}^{\delta} \frac{\psi(t) \sin n t}{t^{2}} d t, \quad I^{\prime}=\int_{\pi / n}^{\delta} \frac{\psi(t) \sin n t}{t(t+\pi / n)} d t,
$$

and $\psi(t)$ satisfies (1.3), then

We have

$$
I-I^{\prime}=o(n), \quad \text { as } n \rightarrow \infty .
$$

$$
\begin{aligned}
I-I^{\prime} & =\frac{\pi}{n} \int_{\pi / n}^{\delta} \frac{\psi(t)}{t^{2}(t+\pi / n)} \sin n t d t \\
& =\frac{\pi}{n}\left[\frac{\Psi(\delta) \sin n \delta}{\delta^{2}(\delta+\pi / n)}-\int_{\pi / n}^{\delta} \Psi(t) \frac{d}{d t}\left\{\frac{\sin n t}{t^{2}(t+\pi / n)}\right\}\right] \\
& =\frac{\pi}{n}\left[O(1)+o\left(\int_{\pi / n}^{\delta} t\left(\frac{n}{t^{3}}+\frac{1}{t^{4}}\right) d t\right)\right] \\
& =o(n)
\end{aligned}
$$

which proves the lemma.

LEMma 2. If $\psi(t)$ satisfies (1.3), then

$$
\int_{\pi / n}^{2 \pi / n} \frac{\psi(t)}{t} \cos n t d t=o(1) \quad \text { as } n \rightarrow \infty .
$$


We have, by integration by parts,

$$
\begin{aligned}
\int_{\pi / n}^{2 \pi / n} \frac{\psi(t)}{t} \cos n t d t & =\left[\Psi(t) \frac{\cos n t}{t}\right]_{\pi / n}^{2 \pi / n}-\int_{\pi / n}^{2 \pi / n} \Psi(t) \frac{d}{d t}\left(\frac{\cos n t}{t}\right) d t \\
& =o(1)+o\left(\int_{\pi / n}^{2 \pi / n}\left(n+\frac{1}{t}\right) d t\right) \\
& =o(1)+o\left([\log t]_{\pi / n}^{2 \pi / n}\right) \\
& =o(1) .
\end{aligned}
$$

Corollary. If

$$
\pi / n \leqq \xi_{1}<\xi_{2} \leqq 2 \pi / n,
$$

then

$$
\int_{\xi_{1}}^{\xi_{2}} \psi(t) \frac{\cos n t}{t} d t=o(1)
$$

and similarly

$$
\int_{\xi_{1}}^{\xi_{2}} \psi(t) \frac{\sin n t}{t} d t=o(1) .
$$

LEMMA $^{1}$ 3. If $\psi(t)$ satisfies (1.3) and (1.4), then

We have

$$
\begin{aligned}
J & =\int_{\pi / n}^{\delta} \frac{\psi(t)}{t(t+\pi / n)} e^{n i t} d t \\
& =o(n) .
\end{aligned}
$$

$$
\begin{aligned}
J & =\left(\int_{\pi / n}^{2 \pi / n}+\int_{2 \pi / n}^{\delta}\right) \frac{\psi(t)}{t(t+\pi / n)} e^{n i t} d t \\
& =J_{1}+J_{2}
\end{aligned}
$$

say. Now by (2.2)

$$
\begin{aligned}
J_{1} & =\frac{n}{2 \pi} \int_{x / n}^{\xi} \frac{\psi(t)}{t} e^{n i t} d t, \quad \frac{\pi}{n}<\xi<\frac{2 \pi}{n}, \\
& =o(n) .
\end{aligned}
$$

Also

1 The proof of this lemma is on the lines of Hardy and Rogosinsky [4] and is given only for the sake of completeness. 


$$
\begin{aligned}
J_{2}= & \int_{2 \pi / n}^{\delta} \frac{\psi(t)}{t(t+\pi / n)} e^{n i t} d t=-\int_{\pi / n}^{\delta-\pi / n} \frac{\psi(t+\pi / n)}{(t+\pi / n)(t+2 \pi / n)} e^{n i t} d t \\
= & -\int_{\pi / n}^{\delta} \frac{\psi(t+\pi / n)}{(t+\pi / n)(t+2 \pi / n)} e^{n i t} d t \\
& +\int_{\delta-\pi / n}^{\delta} \frac{\psi(t+\pi / n)}{(t+\pi / n)(t+2 \pi / n)} e^{n i t} d t \\
(2.6)= & -\int_{\pi / n}^{\delta} \frac{\psi(t+\pi / n)}{(t+\pi / n)(t+2 \pi / n)} e^{n i t} d t+o(1) .
\end{aligned}
$$

From (2.3), (2.4), (2.5) and (2.6) we have

$$
\begin{aligned}
J= & \frac{1}{2} \int_{\pi / n}^{\delta}\left\{\frac{\psi(t)}{t(t+\pi / n)}-\frac{\psi(t+\pi / n)}{(t+\pi / n)(t+2 \pi / n)}\right\} e^{n i t} d t+o(n) \\
= & \frac{1}{2} \int_{\pi / n}^{\delta} \frac{\psi(t)-\psi(t+\pi / n)}{(t+\pi / n)(t+2 \pi / n)} e^{n i t} d t \\
& +\frac{\pi}{n} \int_{\pi / n}^{\delta} \frac{\psi(t)}{t(t+\pi / n)(t+2 \pi / n)} e^{n i t} d t+o(n) \\
= & J_{3}+J_{4}+o(n), \text { say. }
\end{aligned}
$$

Now,

$$
\left|J_{3}\right| \leqq \frac{n}{6 \pi} \int_{\pi / n}^{\delta} \frac{|\psi(t+\pi / n)-\psi(t)|}{t} d t=o(n),
$$

since $\psi(t)$ satisfies (1.4).

Again,

$$
\begin{aligned}
J_{4}= & \frac{\pi}{n} \frac{\Psi(\delta) e^{n i \delta}}{\delta(\delta+\pi / n)(\delta+2 \pi / n)} \\
& -\frac{\pi}{n} \int_{\pi / n}^{\delta} \Psi(t) \frac{d}{d t}\left\{\frac{e^{n i t}}{t(t+\pi / n)(t+2 \pi / n)}\right\} d t \\
= & O\left(\frac{1}{n}\right)+\frac{1}{n} \int_{\pi / n}^{\delta} o(t) \cdot o\left(\frac{n}{t^{3}}+\frac{1}{t^{4}}\right) d t \\
= & o(1)+o(n)=o(n) .
\end{aligned}
$$

Thus

$$
J=o(n)
$$

which proves the lemma. 
3. Proof of the theorem. From Mohanty and Nanda [1], we have

$$
\begin{aligned}
\frac{1}{n} \sum_{1}^{n} r B_{r}(x)-\frac{l}{\pi} & =\frac{1}{\pi} \int_{0}^{\pi}\{f(x+t)-f(x-t)-l\} g(n, t) d t+o(1) \\
& =\frac{1}{\pi} \int_{0}^{\pi} \psi(t) g(n, t) d t+o(1) \\
& =\frac{1}{\pi} P+o(1),
\end{aligned}
$$

say, where

$$
\begin{aligned}
g(n, t) & =-\frac{1}{n} \frac{d}{d t}\{\cos t+\cos 2 t+\cdots+\cos n t\} \\
& =-\frac{1}{n} \frac{d}{d t}\left\{\frac{\cos ((n+1) / 2) t \sin n t / 2}{\sin t / 2}\right\} \\
& =-\frac{1}{2 n} \frac{d}{d t}\left\{\frac{\sin n t}{\tan t / 2}+\cos n t-1\right\} \\
& =\left\{\frac{1}{4 n} \frac{\sin n t}{\sin ^{2} t / 2}-\frac{1}{2} \frac{\cos n t}{\tan t / 2}\right\}+\frac{1}{2} \sin n t
\end{aligned}
$$

Now

$$
\begin{aligned}
P & =\int_{0}^{\pi} \psi(t) g(n, t) d t \\
& =\int_{0}^{\pi} \psi(t)\left[\frac{\sin n t}{4 n \sin ^{2} t / 2}-\frac{\cos n t}{2 \tan t / 2}\right] d t+\frac{1}{2} \int_{0}^{\pi} \psi(t) \sin n t d t \\
& =\int_{0}^{\pi} \psi(t)\left[\frac{\sin n t}{n t^{2}}-\frac{\cos n t}{t}\right] d t+o(1) \\
& =\left(\int_{0}^{\pi / n}+\int_{\pi / n}^{\delta}+\int_{\delta}^{\pi}\right) \psi(t)\left[\frac{\sin n t}{n t^{2}}-\frac{\cos n t}{t}\right] d t+o(1) \\
& =\left(\int_{0}^{\pi / n}+\int_{\pi / n}^{\delta}\right) \psi(t) \sigma(n, t) d t+o(1) \\
(3.2) & =P_{1}+P_{2}+o(1),
\end{aligned}
$$

say, by Riemann Lebesgue Theorem, where

$$
\sigma(n, t)=\frac{\sin n t}{n t^{2}}-\frac{\cos n t}{t}=O\left(n^{2} t\right), \quad \text { as } t \rightarrow 0 .
$$


Now,

$$
\begin{aligned}
P_{1} & =[\Psi(t) \sigma(n, t)]_{0}^{\pi / n}-\int_{0}^{\pi / n} \Psi(t) \frac{d}{d t} \sigma(n, t) d t \\
& =o(1)+o\left(n^{2} \int_{0}^{\pi / n} t d t\right)=o(1)
\end{aligned}
$$

by (1.3).

Again, by applying Lemma 1 and Lemma 2, we have

$$
\begin{aligned}
P_{2} & =\frac{1}{n} \int_{\pi / n}^{\delta} \frac{\psi(t) \sin n t}{t^{2}} d t-\int_{\pi / n}^{\delta} \psi(t) \frac{\cos n t}{t} d t \\
& =\frac{1}{n} \int_{\pi / n}^{\delta} \frac{\psi(t) \sin n t}{t(t+\pi / n)} d t-\int_{\pi / n}^{\delta} \psi(t) \frac{\cos n t}{t} d t+o(1) \\
& =-\int_{\pi / n}^{\delta} \psi(t) \frac{\cos n t}{t} d t+o(1), \quad \text { by }(2.7) \psi(1) \frac{\cos n t}{t} d t+o(1) \\
& =-\left(\int_{\pi / n}^{2 \pi / n}+\int_{2 \pi / n}^{\delta+\pi / n}-\int_{\delta}^{\delta+\pi / n}\right) \psi(\cos n t \\
& =-\int_{2 \pi / n}^{\delta+\pi / n} d t+o(1) \\
& =\int_{\pi / n}^{\delta} \frac{\psi(t+\pi / n) \cos n t}{t+\pi / n} d t+o(1) .
\end{aligned}
$$

By (3.3) and (3.4) we have

$$
\begin{aligned}
\left|P_{2}\right|= & \frac{1}{2}\left|\int_{\pi / n}^{\delta}\left\{\frac{\psi(t+\pi / n)}{t+\pi / n}-\frac{\psi(t)}{t}\right\} \cos n t d t\right|+o(1) \\
= & \frac{1}{2} \mid \int_{\pi / n}^{\delta} \frac{\psi(t+\pi / n)-\psi(t)}{t+\pi / n} \cos n t d t \\
& -\frac{\pi}{2 n} \int_{\pi / n}^{\delta} \frac{\psi(t)}{t(t+\pi / n)} \cos n t d t \mid+o(1) \\
\leqq & \frac{1}{2} \int_{\pi / n}^{\delta} \frac{|\psi(t+\pi / n)-\psi(t)|}{t} d t \\
& +\frac{\pi}{2 n}\left|\int_{\pi / n}^{\delta} \frac{\psi(t)}{t(t+\pi / n)} \cos n t d t\right|+o(1) \\
= & o(1)+\frac{\pi}{2 n} \cdot o(n)=o(1)
\end{aligned}
$$


by (2.7) and (1.4).

From (3.2), (3.3) and (3.6) we obtain

$$
P=o(1) \text {. }
$$

Hence from (3.1) we have

$$
\frac{1}{n} \sum_{1}^{n} r B_{r}(x)-\frac{l}{\pi}=o(1) \quad \text { as } n \rightarrow \infty,
$$

which proves the theorem.

4. Lebesgue's criterion for the convergence of the conjugate series is THEOREM 2. If

$$
\theta(t)=f(x+t)-f(x-t)
$$

satisfies the conditions

$$
\int_{0}^{t} \theta(u) d u=o(t)
$$

and

$$
\int_{\epsilon}^{\delta} \frac{|\theta(t+\epsilon)-\theta(t)|}{t} d t \rightarrow 0
$$

for some fixed $\delta$, when $\epsilon \rightarrow+0$, then the conjugate series $\sum_{1}^{\infty} B_{n}(x)$ converges to the value

$$
\frac{1}{2 \pi} \int_{\rightarrow 0}^{\pi} \theta(t) \cot \frac{t}{2} d t
$$

provided that the integral exists as a Cauchy integral at the origin.

We shall now deduce Theorem 2 as a corollary of Theorem 1 by employing the following

TAuber's SEcond Theorem. If $\sum u_{n}$ is summable (A), then a necessary and sufficient condition that it should be convergent is that the sequence $\left\{n u_{n}\right\}$ is summable $(C, 1)$ to the value zero.

Proof of Theorem 2. The existence of the integral (4.3) as a "Cauchy Integral" at the origin implies the summability (A) of the conjugate series $\sum_{1}^{\infty} B_{n}(x),[3$, p. 55$]$.

By using Theorem 1, we find that conditions (4.1) and (4.2) of Theorem 2 imply the summability $(C, 1)$ of the sequence $\left\{n B_{n}(x)\right\}$ to the value zero. The convergence of the conjugate series then follows from Tauber's Second Theorem. 


\section{REFERENCES}

1. R. Mohanty and M. Nanda, On the behaviour of Fourier coefficients, Proc. Amer. Math. Soc. vol. 5 (1954) pp. 79-84.

2. N. Obrechkoff, Sur la série conjuguée de la serie de Fourier, Annuaire Université de Sofia. I. Faculté Physico-Mathématique. Livre 1, vol. 39 (1943) pp. 321-380.

3 A. Zygmund, Trigonometrical series, 1935, pp. 55, 62.

4. G. H. Hardy and W. W. Rogosinsky, Fourier series, 1944, p. 44.

UNIVERSITY OF SAGAR, INDIA

\section{A NOTE ON HAUSDORFF TRANSFORMS}

\section{AMNON JAKIMOVSKI}

1. Introduction. If $\left\{\mu_{p}\right\}(p=0,1,2, \cdots)$ is an arbitrary sequence of numbers, we shall define the double sequence $\left(\Delta^{n} \mu_{p}\right)$ $(n, p=0,1,2, \cdots)$ by

$$
\Delta^{n} \mu_{p}=\sum_{r=0}^{n}(-1)^{r} \cdot\left(\begin{array}{l}
n \\
r
\end{array}\right) \cdot \mu_{p+r} \quad(n, p=0,1,2, \cdots) .
$$

The quantities (1.1) are generally referred to as the "differences" of the sequence $\left\{\mu_{p}\right\}$ since they satisfy the recurrence relations

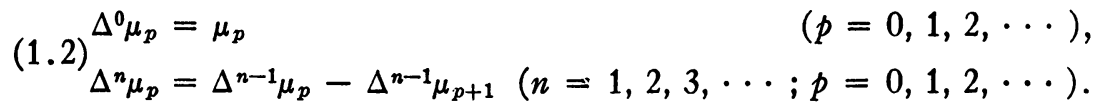

In this paper, we shall say that the sequence $\left\{t_{p}\right\}$ is the Hausdorff transform of the sequence $\left\{s_{p}\right\}$ generated by the sequence $\left\{\mu_{p}\right\}$ in case

$$
t_{p}=\sum_{q=0}^{p}\left(\begin{array}{l}
p \\
q
\end{array}\right) \cdot\left(\Delta^{p-q} \mu_{q}\right) \cdot s_{q} \quad(p=0,1,2, \cdots) .
$$

A Hausdorff transform generated by a sequence $\left\{\mu_{p}\right\}$ will be called here, for shortness, an $\left(H, \mu_{p}\right)$ transform.

Although transformations of the form (1.3) are generally referred to as Hausdorff transformations, it is to be noted that they were actually first investigated to any extent by Hurwitz and Silverman $[1],{ }^{1}$ who characterized them as sequence-to-sequence transformations generated by triangular matrices $A$ permutable with the matrix

Received by the editors May 9, 1955 and, in revised form, October 24, 1955.

${ }^{1}$ Numbers in square brackets refer to references at the end of this note. 